\title{
Distributions of several infinite families of mesh patterns
}

\author{
Sergey Kitaev ${ }^{a}$, Philip B. Zhang ${ }^{b}$, Xutong Zhang ${ }^{c}$ \\ ${ }^{a}$ Department of Computer and Information Sciences \\ University of Strathclyde, 26 Richmond Street, Glasgow G1 1XH, UK \\ ${ }^{b, c}$ College of Mathematical Science \\ Tianjin Normal University, Tianjin 300387, China \\ Email: ${ }^{a}$ sergey.kitaev@cis.strath.ac.uk, ${ }^{b}$ zhang@tjnu.edu.cn, \\ ${ }^{c}$ zhang.xutong@foxmail.com
}

\begin{abstract}
Brändén and Claesson introduced mesh patterns to provide explicit expansions for certain permutation statistics as linear combinations of (classical) permutation patterns. The first systematic study of the avoidance of mesh patterns was conducted by Hilmarsson et al., while the first systematic study of the distribution of mesh patterns was conducted by the first two authors.

In this paper, we provide far-reaching generalizations for 8 known distribution results and 5 known avoidance results related to mesh patterns by giving distribution or avoidance formulas for certain infinite families of mesh patterns in terms of distribution or avoidance formulas for smaller patterns. Moreover, as a corollary to a general result, we find the distribution of one more mesh pattern of length 2 .
\end{abstract}

Keywords: mesh pattern, distribution, avoidance

AMS Subject Classifications: 05A15

\section{Introduction}

Patterns in permutations and words have attracted much attention in the literature (see [6] and references therein), and this area of research continues to grow rapidly. The notion of a mesh pattern, generalizing several classes of patterns, was introduced by Brändén and Claesson [3] to provide explicit expansions for certain permutation statistics as, possibly infinite, linear combinations of (classical) permutation patterns. Several papers are dedicated to the study of mesh patterns and their generalizations $[1,2,5,7,8,9,13,14]$. 
Let $\llbracket 0, k \rrbracket$ denote the interval of the integers from 0 to $k$. A pair $(\tau, R)$, where $\tau$ is a permutation of length $k$ written in one-line notation and $R$ is a subset of $\llbracket 0, k \rrbracket \times \llbracket 0, k \rrbracket$, is a mesh pattern of length $k$. Let $(i, j)$ denote the box whose corners have coordinates $(i, j),(i, j+1),(i+1, j)$, and $(i+1, j+1)$. Let the horizontal lines represent the values, and the vertical lines denote the positions in the pattern. Mesh patterns can be drawn by shading the boxes in $R$. For example, the picture

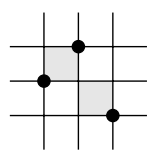

represents the mesh pattern with $\tau=231$ and $R=\{(1,2),(2,1)\}$. A mesh pattern $(\tau, R)$ of length $k \geq 2$ is irreducible if the permutation $\tau=\tau_{1} \tau_{2} \cdots \tau_{k}$ is irreducible, that is, if there exists no $i$, where $2 \leq i \leq k$, such that $\tau_{j}<\tau_{i}$ for all $1 \leq j<i$. For convenience, in this paper we assume that if $\tau$ is of length 1 then it is not irreducible, even though normally such a $\tau$ is assumed to be irreducible. All mesh patterns of interest in this paper can be found in Tables $1-3$, where patterns' numbers $<66$ are coming from $[4,10]$. Also, we let $Z:=\rightarrow-$

A subsequence $\pi^{\prime}=\pi_{i_{1}} \pi_{i_{2}} \cdots \pi_{i_{k}}$ of a permutation $\pi=\pi_{1} \pi_{2} \cdots \pi_{n}$ is an occurrence of a mesh pattern $(\tau, R)$ if (a) $\pi^{\prime}$ is order-isomorphic to $\tau$, and (b) the shaded squares given by $R$ do not contain any elements of $\pi$ not appearing in $\pi^{\prime}$. For example, the mesh pattern of length 3 drawn above appears twice in the permutation 24531 (as the subsequences 241 and 453). Note that even though the subsequences 251 and 451 are order isomorphic to 231 (the $\tau$ in the drawn pattern), they are not occurrences of the pattern because of the elements 4 and 3, respectively, be in the shaded squares. See [4] for more examples of occurrences of mesh patterns in permutations.

Let $S_{n}$ be the set of permutations of length $n$. Given a permutation $\pi$, denote by $p(\pi)$ the number of occurrences of pattern $p$ in $\pi$. Denote by $S(p)$ set of permutations avoiding $p$. We let $A_{p}(x)$ be the generating function for $S(p)$ and let

$$
F_{p}(x, q):=\sum_{n \geq 0} x^{n} \sum_{\pi \in S_{n}} q^{p(\pi)} .
$$

In this paper we provide various generalizations of results in [10]. The main idea of this paper is to consider a mesh pattern $p$, and to replace some of its unshaded boxes by mesh patterns. To illustrate this idea, consider the mesh pattern 


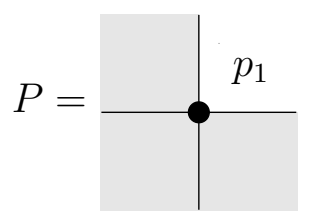

obtained from the smaller mesh pattern $Y=\downarrow$ by inserting a mesh pattern $p_{1}$ in the box $(1,1)$. We can then find the distribution of $P$ in terms of the distribution of $p_{1}$, which not only allows us to obtain three results in [10] at the same time (distributions of the patterns Nr. 12, 13, and 17; see Section 2) but also to derive the previously unknown distribution of the pattern

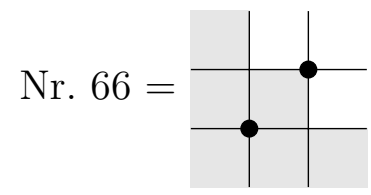

(pattern's number is introduced by us in this paper) which is not equivalent to any of the patterns in [4].

For a more sophisticated example illustrating the power of our results in this paper, suppose that one wants to find the distribution of the mesh pattern in Figure 1. Approaching this problem directly is probably not doable. However, one can see that the elements $a$ and $b$ give a mesh pattern of the form in Figure 4, so that Theorem 3.2 can be applied with $p_{1}$ there given by the elements $c$ and $d$, which give a mesh pattern of the form in Figure 7, so that Theorem 3.5 can be applied. Finally, $p_{1}$ in Theorem 3.5 in our example is nothing else but the mesh pattern Nr. 66, so Corollary 2.4 can be applied. This will result in the distribution of the mesh pattern in Figure 1 be

$F(x)\left(1+x(F(x)-1)\left(\frac{1}{1+x^{2} F(x)\left(F(x)-1-q x \sum_{n=1}^{\infty} \prod_{i=1}^{n-1}(q+i) x^{n}\right)}-1\right)\right)$,

where $F(x):=\sum_{n \geq 0} n ! x^{n}$.

In Tables 1 and 2 we give references to our enumerative results related to distribution and avoidance, respectively. Moreover, in Table 3 we give references to our distribution and avoidance results on certain generalizations of short mesh patterns. We note that the patterns $p_{1}, p_{2}, p_{3}$ in Tables 1-3 can be empty, in which case one needs to substitute the generating functions $A_{p_{i}}(x)$ and $F_{p_{i}}(x, q)$ by 0 and $q F(x)$, respectively, in our results. Indeed, one can assume that any permutation contains exactly one occurrence of the empty pattern, which makes the substitutions work. Also, in the case of 


\begin{tabular}{|c|c|c|c|c|c|c|}
\hline Nr. & Repr. $p$ & Generalization & $p_{1}$ & $p_{2}$ & $p_{3}$ & Distribution \\
\hline$X$ & $\rightarrow$ & $\bullet^{p_{1}}$ & Irreducible & - & - & Theorem 4.1 \\
\hline$Y$ & $\rightarrow-$ & $\bullet^{p_{1}}$ & Any & - & - & Theorem 2.1 \\
\hline 12 & $\doteqdot$ & - & - & - & - & Corollary 2.2 \\
\hline 13 & $\because \div$ & $\int_{i}^{p_{i}}$ & Any & - & - & $\begin{array}{l}\text { Theorem } 3.1 \\
\text { Corollary } 2.3\end{array}$ \\
\hline 17 & $\doteqdot$ & - & - & - & - & Corollary 2.4 \\
\hline 19 & $\stackrel{\square}{\oplus}$ & $\cdot^{p_{1}}$ & Any & - & - & Theorem 3.2 \\
\hline 20 & $\because+$ & ${\stackrel{\cdot}{p_{2}}}^{p_{1}}$ & Any & Any & - & Theorem 3.3 \\
\hline 22 & $\because$ & $\begin{array}{l}p_{1} \mid \\
\cdot p_{p_{3}} \\
p_{p_{2}}\end{array}$ & Any & Any & Any & Theorem 3.4 \\
\hline 28 & $\stackrel{4}{\ddagger}$ & $\int_{1}^{p_{1}^{\natural}}$ & Any & - & - & Theorem 3.5 \\
\hline 33 & 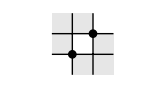 & ${\stackrel{\bullet}{i^{p_{1}}}}^{p^{1}}$ & Irreducible & - & - & Theorem 4.2 \\
\hline 66 & 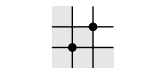 & - & - & - & - & Corollary 2.5 \\
\hline
\end{tabular}

Table 1: Distributions of generalizations of short mesh patterns. Note that pattern $X$ is essentially equivalent to pattern $Z$ in the sense of distribution. 


\begin{tabular}{|c|c|c|c|c|c|}
\hline Nr. & Repr. $p$ & Generalization & $p_{1}$ & $p_{2}$ & Avoidance \\
\hline 27 & $\because$ & ${ }_{\overrightarrow{p_{1}}}^{\mathfrak{p}^{p_{2}}}$ & Any & Any & Theorem 5.5 \\
\hline 28 & $\because$ & $\frac{p^{p_{1}}}{{\stackrel{0}{p_{2}}}^{2}}$ & Any & Any & Theorem 5.1 \\
\hline 30 & $\because$ & ${\stackrel{\cdot}{\bullet^{p_{1}}}}^{-}$ & Two general classes & - & Theorem 5.4 \\
\hline
\end{tabular}

Table 2: Avoidance for generalizations of short mesh patterns.

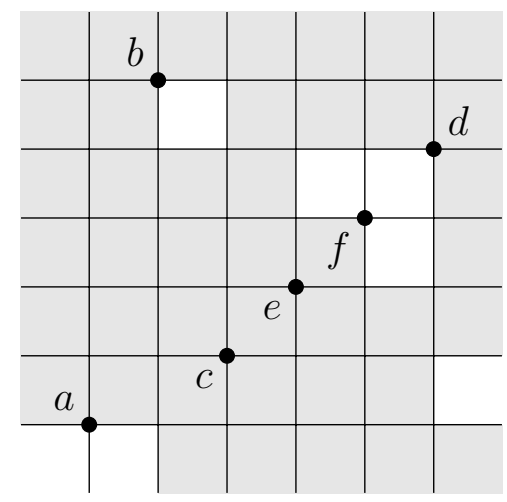

Figure 1: A mesh pattern of length 6

empty $p_{1}$, one needs to set $k=1$ in our results related to the pattern Nr. 34 (in Theorems 5.7 and 5.8). In this way, one can obtain any previous results in $[4,10]$ related to the patterns appearing in this paper.

In this paper, we need the following result.

Theorem 1.1 ([10, Theorem 1.1]). Let

$$
F(x, q)=\sum_{n \geq 0} x^{n} \sum_{\pi \in S_{n}} q^{\dagger(\pi)}=\sum_{n \geq 0} x^{n} \sum_{\pi \in S_{n}} q^{\dagger(\pi)}
$$

and let $A(x)$ be the generating function for $S(+)=S(+)$. Then,

$$
A(x)=\frac{F(x)}{1+x F(x)}, \quad F(x, q)=\frac{F(x)}{1+x(1-q) F(x)} .
$$




\begin{tabular}{|c|c|c|c|c|c|}
\hline Nr. & Repr. $p$ & Generalization & $p_{1}$ & $p_{2}$ & Reference \\
\hline 33 & $\because$ & $?^{p_{1}^{p_{1}}}$ & Irreducible & - & Theorem 4.3 \\
\hline 34 & $\because \bullet$ & ${ }_{i}^{p_{1}}$ & Any & - & Theorem 5.7 \\
\hline 34 & $\div$ & $\frac{p_{1}}{p_{1}}$ & Any & Any & Theorem 5.8 \\
\hline
\end{tabular}

Table 3: Generalizations of short mesh patterns allowing replacement of $\tau=12$ either by an increasing permutation or by a permutation beginning with the smallest element with all boxes shaded. The distributions are given for Nr. 33, and 34 with $p_{1}$ but without $p_{2}$, and the avoidance for Nr. 34 with $p_{1}$ and $p_{2}$.

This paper is organized as follows. In Section 2 we present distribution and avoidance results for certain mesh patterns derived from the pattern $Y$. In Section 3 we study the distribution and avoidance for certain patterns derived from the patterns Nr. 13, 19, 20, 22, and 28. Section 4 is dedicated to the distribution and avoidance for certain mesh patterns derived from the pattern $X$. In Section 5 we deal with avoidance results for certain mesh patterns derived from the patterns Nr. 27, 28, 30, and 34. Finally, we provide some concluding remarks in Section 6.

\section{A generalization of the pattern $Y$}

In this section, we consider a generalization of the pattern $Y=\downarrow$. As an application of our general results, we will find the distributions of the following patterns:

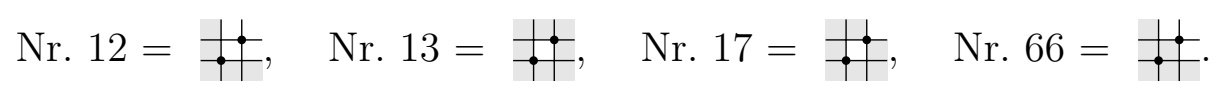

Theorem 2.1. Suppose that $p$ is the pattern shown in Figure 2, where $p_{1}$ is 
any mesh pattern, and the label $a$ is to be ignored. Then,

$$
\begin{aligned}
A_{p}(x) & =(1-x) F(x)+x A_{p_{1}}(x), \\
F_{p}(x, q) & =(1-x) F(x)+x F_{p_{1}}(x, q) .
\end{aligned}
$$

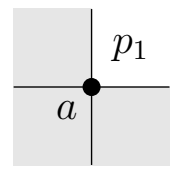

Figure 2: Related to the proof of Theorem 2.1.

Proof. Any permutation counted by $F(x)$ either avoids $p$, which is counted by $A_{p}(x)$, or contains at least one occurrence of $p$. The generating function for the latter case is $x\left(F(x)-A_{p_{1}}(x)\right)$. Indeed, for any occurrence of $p$, the element $a$ in Figure 2 is the same. Now the North East box in the figure must contain at least one occurrence of $p_{1}$, which is counted by $F(x)-A_{p_{1}}(x)$, and the element $a$ contributes the factor of $x$. This leads to

$$
A_{p}(x)+x\left(F(x)-A_{p_{1}}(x)\right)=F(x) .
$$

For the distribution, we have the following functional equation:

$$
A_{p}(x)+x\left(F_{p_{1}}(x, q)-A_{p_{1}}(x)\right)=F_{p}(x, q) .
$$

Our proof of (4) is essentially the same as that in the avoidance case. In particular, the contribution of the North East box is $F_{p_{1}}(x, q)-A_{p_{1}}(x)$, since every occurrence of $p_{1}$ there, along with the element $a$, will give an occurrence of $p$, and all occurrences of $p$ are obtained in this way. The formulas (1) and (2) now follow from the formulas (3) and (4), respectively. This completes the proof.

The following results follow from Theorem 2.1.

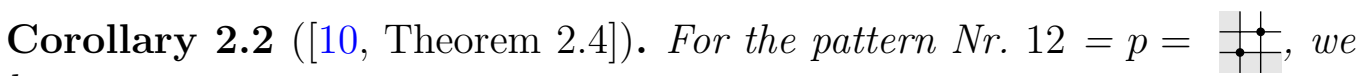
have

$$
\begin{aligned}
A_{p}(x) & =(1-x) F(x)+x, \\
F_{p}(x, q) & =(1-x) F(x)+x F(q x) .
\end{aligned}
$$


Proof. The mesh pattern $p$ is obtained from the pattern $Y$ by inserting the pattern $p_{1}=\rightarrow$. It is easy to see that $A_{p_{1}}(x)=1$ and $F_{p_{1}}(x, q)=F(q x)$. After substituting these into (1) and (2), we obtain the desired result.

Corollary 2.3 ([10, Theorem 2.5]). For the pattern Nr. $13=p=! \cdot$, we have

$$
\begin{aligned}
A_{p}(x) & =\left(1-x^{2}\right) F(x), \\
F_{p}(x, q) & =\left(1-x^{2}+q x^{2}\right) F(x) .
\end{aligned}
$$

Proof. The mesh pattern $p$ is obtained from the pattern $Y$ by inserting the pattern $p_{1}=\bullet$. It is easy to see that $A_{p_{1}}(x)=1$ and $F_{p_{1}}(x, q)=F(q x)$. Together with $A_{p_{1}}(x)=F(x)-x F(x)$, and $F_{p_{1}}(x, q)=F(x)-x F(x)+$ $q x F(x)$, we have the desired result.

Corollary 2.4 ([10, Theorem 3.2]). For the pattern Nr. $17=p=\frac{!}{\dagger}$, we have

$$
F_{p}(x, q)=\left(1-x+\frac{x}{1+x(1-q) F(x)}\right) F(x) .
$$

Also, $A_{p}(x)=F_{p}(x, 0)$.

Proof. The mesh pattern $p$ is obtained from the pattern $Y$ by inserting the pattern $p_{1}=\downarrow$. By Theorem 1.1, we have $F_{p_{1}}(x, q)=\frac{F(x)}{1+x(1-q) F(x)}$. After substituting it into (2), we obtain the desired result.

The following result is new.

Corollary 2.5. For the pattern Nr. $66=p=\ddagger !$, we have

$$
\begin{aligned}
A_{p}(x) & =(1-x) F(x)+x \\
F_{p}(x, q) & =(1-x) F(x)+x+x \sum_{n=1}^{\infty} \prod_{i=0}^{n-1}(q+i) x^{n} .
\end{aligned}
$$

Proof. The mesh pattern $p$ is obtained from the pattern $Y$ by inserting the pattern $p_{1}=\bullet-$. Note that $A_{p_{1}}(x)=1$ and the distribution of $p_{1}$ is given by the unsigned Stirling numbers of the first kind [12, p. 19]:

$$
F_{p_{1}}(x, q)=1+\sum_{n=1}^{\infty} \prod_{i=0}^{n-1}(q+i) x^{n} .
$$

Substituting these into (1) and (2) gives the desired result. 


\section{Distributions of several mesh patterns}

In this section, we generalize known distribution results for the patterns

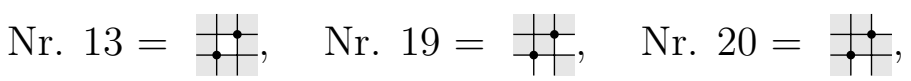

$$
\begin{aligned}
& \text { Nr. } 22=\underset{\dagger}{\dagger}, \quad \text { Nr. } 28=\underset{\dagger}{\dagger} \text {. }
\end{aligned}
$$

The theorems proved in this section are not so difficult (and somewhat similar), but they prepare the reader for the upcoming more involved distribution or avoidance results.

\subsection{The pattern Nr. 13}

We first consider the generalization of the pattern Nr. $13=\underset{\rightarrow}{ }$.

Theorem 3.1. Suppose that $p$ is the pattern shown in Figure 3, where $p_{1}$ is any mesh pattern, and the labels $a$ and $b$ are to be ignored. Then,

$$
\begin{aligned}
A_{p}(x) & =F(x)-x^{2}\left(F(x)-A_{p_{1}}(x)\right), \\
F_{p}(x, q) & =\left(1-x^{2}\right) F(x)+x^{2} F_{p_{1}}(x, q) .
\end{aligned}
$$

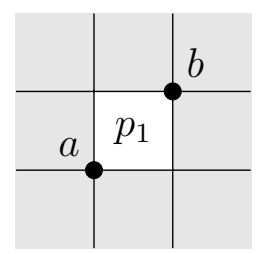

Figure 3: Related to the proof of Theorem 3.1.

Proof. We have the following functional equation:

$$
A_{p}(x)+x^{2}\left(F(x)-A_{p_{1}}(x)\right)=F(x) .
$$

Indeed, any permutation counted by $F(x)$ either avoids $p$, which is counted by $A_{p}(x)$, or contains at least one occurrence of $p$. The generating function for the latter case is $x^{2}\left(F(x)-A_{p_{1}}(x)\right)$. Indeed, an occurrence of $p$ implies at 
least one occurrence of $p_{1}$ in the central box in Figure 3, which contributes the factor of $F(x)-A_{p_{1}}(x)$. Besides, the factor of $x^{2}$ is contributed by $a b$.

For the distribution, we have the following functional equation:

$$
A_{p}(x)+x^{2}\left(F_{p_{1}}(x, q)-A_{p_{1}}(x)\right)=F_{p}(x, q) .
$$

The proof of (6) is essentially the same as that in the avoidance case. In particular, the factor of $F_{p_{1}}(x, q)-A_{p_{1}}(x)$ comes from the fact that in any occurrence of $p, a$, and $b$ in Figure 3 must be the same. The desired result follows from (5) and (6). This completes the proof.

\subsection{The pattern Nr. 19}

Now we generalize the pattern Nr. $19=\underset{\dagger}{\dagger}$.

Theorem 3.2. Suppose that $p$ is the pattern shown in Figure 4 , where $p_{1}$ is any mesh pattern, and the labels $a, b, A$, and $B$ are to be ignored. Then,

$$
\begin{gathered}
A_{p}(x)=F(x)-x(F(x)-1)\left(F(x)-A_{p_{1}}(x)\right), \\
F_{p}(x, q)=F(x)+x(F(x)-1)\left(F_{p_{1}}(x, q)-F(x)\right) .
\end{gathered}
$$

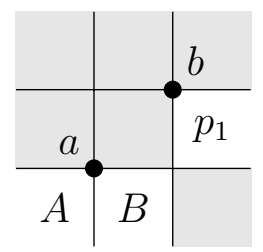

Figure 4: Related to the proof of Theorem 3.2.

Proof. We have the following functional equation:

$$
A_{p}(x)+x(F(x)-1)\left(F(x)-A_{p_{1}}(x)\right)=F(x),
$$

where the right hand side counts all permutations. Indeed, each permutation either avoids $p$, which is given by the $A_{p}(x)$ term in $(7)$, or it contains at least one occurrence of $p$. The latter case is given by the second term on the left hand side of (7), which shall be proved in the following. To pick 
the occurrence $a b$ in Figure 4, we choose $b$ the highest possible and $a$ is then uniquely determined. Referring to this figure, we note that the East box must contain at least one occurrence of $p_{1}$, which is counted by $F(x)-A_{p_{1}}(x)$. Furthermore, the boxes $A$ and $B$ together with $a$, which is the maximum element in the permutation $A a B$, contribute the factor of $F(x)-1$. Note that $a$ must exist, so $A a B$ is not empty and there are no other restrictions for $A a B$. Finally, $b$ contributes the factor of $x$. Thus, we complete the proof of (7) and hence give the formula of $A_{p}(x)$.

For the distribution, we have the following functional equation:

$$
A_{p}(x)+x(F(x)-1)\left(F_{p_{1}}(x, q)-A_{p_{1}}(x)\right)=F_{p}(x, q) .
$$

The proof of (8) is essentially the same as that in the avoidance case. Since $a$ is uniquely determined, the boxes $A$ and $B$ together with $a$ contribute the factor of $F(x)-1$. The East box contributes the factor of $F_{p_{1}}(x, q)-A_{p_{1}}(x)$, since each occurrence of $p_{1}$ in that box induces one occurrence of $p$. Finally, the factor of $x$ corresponds to the element $b$, which completes the proof of (8). Substituting the formula of $A_{p}(x)$ into (8) gives the formula of $F_{p}(x, q)$ as desired.

\subsection{The pattern $\mathrm{Nr} .20$}

Now, we generalize the pattern $\underset{\dagger}{\leftrightarrows}$ by adding $p_{1}$ and $p_{2}$ in it.

Theorem 3.3. Suppose that $p$ is the pattern shown in Figure 5, where $p_{1}$ and $p_{2}$ are any mesh patterns, and the labels a and $b$ are to be ignored. Then,

$$
\begin{aligned}
A_{p}(x)= & F(x)-x^{2}\left(F(x)-A_{p_{1}}(x)\right)\left(F(x)-A_{p_{2}}(x)\right), \\
F_{p}(x, q)= & F(x)+x^{2}\left(\left(F_{p_{1}}(x, q)-A_{p_{1}}(x)\right)\left(F_{p_{2}}(x, q)-A_{p_{2}}(x)\right)-\right. \\
& \left.\left(F(x)-A_{p_{1}}(x)\right)\left(F(x)-A_{p_{2}}(x)\right)\right) .
\end{aligned}
$$

Proof. We have the following functional equation:

$$
A_{p}(x)+x^{2}\left(F(x)-A_{p_{1}}(x)\right)\left(F(x)-A_{p_{2}}(x)\right)=F(x) .
$$

Indeed, the right hand side of (9) counts all permutations. On the left hand side, $A_{p}(x)$ gives avoidance of $p$, and the other term, to be justified next, counts permutations with at least one occurrence of $p$. Indeed, the elements 


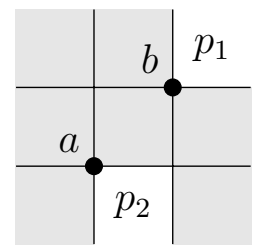

Figure 5: Related to the proof of Theorem 3.3.

$a$ and $b$ contributing the factor of $x^{2}$ are uniquely determined, which is evident from Figure 5. Referring to this figure, we note that the upper (resp., lower) non-shaded box $A$ (resp., $B$ ) must contain at least one occurrence of $p_{1}$ (resp., $p_{2}$ ) counted by $F(x)-A_{p_{1}}(x)$ (resp., $\left.F(x)-A_{p_{2}}(x)\right)$. Thus, we complete the proof of (9) and hence give the formula of $A_{p}(x)$.

For the distribution, we have the following functional equation:

$$
A_{p}(x)+x^{2}\left(F_{p_{1}}(x, q)-A_{p_{1}}(x)\right)\left(F_{p_{2}}(x, q)-A_{p_{2}}(x)\right)=F_{p}(x, q) .
$$

The proof of (10) is essentially the same as that in the avoidance case. The box $A$ (resp., $B$ ) contributes the factor of $F_{p_{1}}(x, q)-A_{p_{1}}(x)$ (resp., $F_{p_{2}}(x, q)-$ $\left.A_{p_{2}}(x)\right)$, since each occurrence of $p_{1}$ in $A$, together with any occurrence of $p_{2}$ in $B$, form an occurrence of $p$. Together with the factor of $x^{2}$ corresponding to $a b$, we complete the proof of (10). Substituting the formula of $A_{p}(x)$ into (10) gives the desired result.

\subsection{The pattern Nr. 22}

Next we generalize the pattern $\biguplus^{\leftarrow}$ by adding $p_{1}, p_{2}$, and $p_{3}$ in it.

Theorem 3.4. Suppose that $p$ is the pattern shown in Figure 6, where $p_{1}$, $p_{2}$, and $p_{3}$ are any mesh patterns, and the labels $a$ and $b$ are to be ignored. Then,

$$
\begin{gathered}
A_{p}(x)=F(x)-x^{2}\left(F(x)-A_{p_{1}}(x)\right)\left(F(x)-A_{p_{2}}(x)\right)\left(F(x)-A_{p_{3}}(x)\right) \\
F_{p}(x, q)=F(x)+x^{2}\left(\left(F_{p_{1}}(x, q)-A_{p_{1}}(x)\right)\left(F_{p_{2}}(x, q)-A_{p_{2}}(x)\right)\left(F_{p_{3}}(x, q)-A_{p_{3}}(x)\right)\right. \\
\left.\quad-\left(F(x)-A_{p_{1}}(x)\right)\left(F(x)-A_{p_{2}}(x)\right)\left(F(x)-A_{p_{3}}(x)\right)\right) .
\end{gathered}
$$




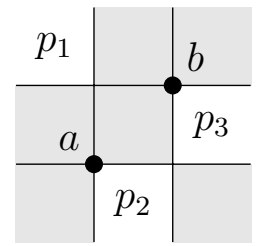

Figure 6: Related to the proof of Theorem 3.4.

Proof. Any permutation, counted by $F(x)$, either avoids $p$, which is counted by $A_{p}(x)$, or contains at least one occurrence of $p$. In the latter situation, the elements $a b$ in an occurrence of $p$ are uniquely defined. Indeed, looking at Figure 6 we see that another occurrence of $p$ cannot be inside of any of the unshaded boxes:

- if $p$ would occur in the box labeled with $p_{1}$, it would contradict the South East box being shaded;

- if $p$ would occur in the box labeled with $p_{2}$, it would contradict the element $b$ being in the North East shaded box;

- if $p$ would occur in the box labeled with $p_{3}$, it would contradict the element $a$ being in the South West shaded box.

Moreover, another occurrence of $p$ cannot begin at the box

- labeled $p_{1}$ because of the two shaded top boxes;

- labeled $p_{2}$ because of the element $a$;

- labeled $p_{3}$ because of the North East box being shaded.

So, $a$ and $b$ will contribute the factor of $x^{2}$. Referring to this figure, we note that the non-shaded box labeled by $p_{1}$ (resp., $p_{2}$ and $p_{3}$ ) must contain at least one occurrence of $p_{1}$ (resp., $p_{2}$ and $p_{3}$ ) and thus is counted by $F(x)-A_{p_{1}}(x)$ (resp., $F(x)-A_{p_{2}}(x)$ and $\left.F(x)-A_{p_{3}}(x)\right)$. Thus, we obtain

$$
A_{p}(x)+x^{2}\left(F(x)-A_{p_{1}}(x)\right)\left(F(x)-A_{p_{2}}(x)\right)\left(F(x)-A_{p_{3}}(x)\right)=F(x),
$$

which leads to the formula of $A_{p}(x)$. 
For the distribution, we have the following functional equation:

$$
\begin{gathered}
A_{p}(x)+x^{2}\left(F_{p_{1}}(x, q)-A_{p_{1}}(x)\right)\left(F_{p_{2}}(x, q)-A_{p_{2}}(x)\right)\left(F_{p_{3}}(x, q)-A_{p_{3}}(x)\right) \\
=F_{p}(x, q) .
\end{gathered}
$$

The proof of (11) is essentially the same as that in the avoidance case. The only remark is that any occurrence of the pair $\left(p_{1}, p_{2}, p_{3}\right)$ in their respective non-shaded boxes induce an occurrence of $p$. Substituting the formula of $A_{p}(x)$ into (11) gives the formula of $F_{p}(x, q)$. This completes the proof.

\subsection{The pattern Nr. 28}

We now generalize the pattern $\underset{\ddagger}{\bullet}$ by adding $p_{1}$ in it.

Theorem 3.5. Suppose that $p$ is the pattern shown in Figure \%, where $p_{1}$ is any mesh pattern, and the labels $a, b, A$, and $B$ are to be ignored. Then,

$$
\begin{aligned}
A_{p}(x) & =\frac{F(x)}{1+x^{2}\left(F(x)-A_{p_{1}}(x)\right) F(x)}, \\
F_{p}(x, q) & =\frac{F(x)}{1+x^{2}\left(F(x)-F_{p_{1}}(x, q)\right) F(x)} . \\
&
\end{aligned}
$$

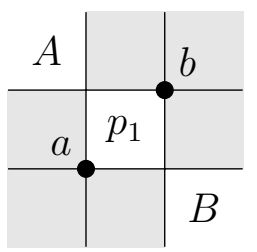

Figure 7: Related to the proof of Theorem 3.5.

Proof. Similar to the avoidance case of Theorem 3.2, we have

$$
A_{p}(x)+x^{2} A_{p}(x)\left(F(x)-A_{p_{1}}(x)\right) F(x)=F(x) .
$$

The only term requiring an explanation here is $x^{2} A_{p}(x)\left(F(x)-A_{p_{1}}(x)\right) F(x)$ corresponding to permutations with at least one occurrence of $p$. Among all such occurrences consider $a b$ with leftmost possible $a$ as shown in Figure 7. 
Note that the element $b$ is then uniquely determined. Further, the middle box must contain at least one occurrence of $p_{1}$, which is counted by $F(x)-A_{p_{1}}(x)$, and the permutation in box $A$ must be $p$-avoiding since $a$ is the leftmost. Moreover, box $B$ can contain any permutation, and thus its contribution is $F(x)$. Finally, $a$ and $b$ contribute the factor of $x^{2}$, which completes the proof of (12) and hence gives the formula of $A_{p}(x)$.

For the distribution, we have the following functional equation:

$$
A_{p}(x)+x^{2} A_{p}(x)\left(F_{p_{1}}(x, q)-A_{p_{1}}(x)\right) F_{p}(x, q)=F_{p}(x, q) .
$$

The proof of (13) is essentially the same as that in the avoidance case. We just remark that any occurrence of $p_{1}$ in the middle box gives an occurrence of $p$, which explains the term $F_{p_{1}}(x, q)-A_{p_{1}}(x)$, and $F_{p}(x, q)$ on the left hand side of (13) records occurrences of $p$ in box $B$.

\section{Patterns derived from the pattern $X$}

This section gives the distribution of an infinite family of mesh patterns obtained from the pattern $X=\bullet \bullet$. Namely, we generalize $X$ by considering $\mathfrak{i}^{p_{1}}$, where $p_{1}$ is an irreducible mesh pattern. Moreover, we use the same approach to study the distribution of a family of patterns that can be derived from the pattern Nr. $33=\underset{\dagger}{ }$. Such distributions cannot be directly obtained from our results for $\mapsto_{\bullet}^{p_{1}}$, since $p_{1}$ must be irreducible there.

\subsection{The pattern $X$}

In our next theorem we consider a generalization of $X$.

Theorem 4.1. Suppose that $p$ is the pattern shown in Figure 8, where $p_{1}$ is an irreducible mesh pattern of length $\geq 2$, and the label a is to be ignored. Then,

$$
\begin{aligned}
A_{p}(x) & =\frac{\left(1+x A_{p_{1}}(x)\right) F(x)}{1+x F(x)} \\
F_{p}(x, q) & =\left(1+\sum_{i \geq 1} x^{i} \prod_{j=1}^{i} \frac{F_{p_{1}}\left(x, q^{j}\right)}{1+x F_{p_{1}}\left(x, q^{j}\right)}\right) \frac{F(x)}{1+x F(x)} .
\end{aligned}
$$




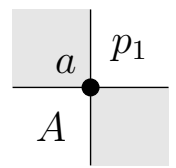

Figure 8: Related to the proof of Theorem 4.1.

Proof. Let $B(x)$ be the generating function for $X$-avoiding permutations. Then, it follows from Theorem 1.1 that

$$
B(x)=\frac{F(x)}{1+x F(x)} .
$$

We next justify the following functional equation:

$$
A_{p}(x)+x B(x)\left(F(x)-A_{p_{1}}(x)\right)=F(x) .
$$

The right hand side counts all permutations. On the left hand side, $A_{p}(x)$ counts $p$-avoiding permutations. If a permutation contains at least one occurrence of $p$, we can consider the occurrence with the leftmost possible $a$ as shown in Figure 8. Referring to this figure, we note that the Noth East box must contain at least one occurrence of $p_{1}$, which is counted by $F(x)-A_{p_{1}}(x)$, and a permutation in box $A$ must be $\downarrow-$-avoiding since $a$ is the leftmost. Finally, $a$ contributes the factor of $x$. This completes the proof of (15). Substituting (14) into (15), we obtain the formula of $A_{p}(x)$.

In order to study the distribution of $p$, we also need the distribution of $p_{1}$. Let $B_{p_{1}}(x, q)$ be the distribution of $p_{1}$ on $X$-avoiding permutations. Then,

$$
B_{p_{1}}(x, q)+x B_{p_{1}}(x, q) F_{p_{1}}(x, q)=F_{p_{1}}(x, q) .
$$

This equation is obtained by considering $X$-avoiding permutations separately from the other permutations, and only the second term on the left hand side, corresponding to permutations with at least one occurrence of $X$, requires a justification. Consider the occurrence of $X$ with the leftmost $a$ as in Figure 8. Any permutation in box $A$ is $X$-avoiding and thus contributes the factor of $B_{p_{1}}(x, q)$. Since $p_{1}$ is irreducible, the two non-shaded boxes in Figure 8 are independent from each other. In other words, an occurrence of $p_{1}$ cannot start in $A$ and end in the other non-shaded box. Thus, the contribution of the North East box is the factor of $F_{p_{1}}(x, q)$ in $(16)$. This, along with the 
factor of $x$ corresponding to $a$, completes the proof of (16). Therefore, it follows from (16) that

$$
B_{p_{1}}(x, q)=\frac{F_{p_{1}}(x, q)}{1+x F_{p_{1}}(x, q)} .
$$

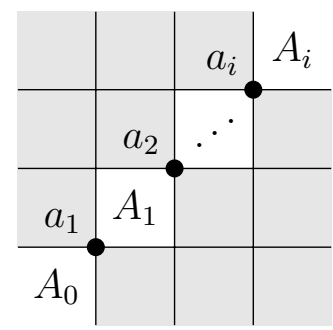

Figure 9: Related to the proof of Theorem 4.1.

Now we consider the distribution of $p$. Claim that

$$
B(x)+B(x) \sum_{i \geq 1} x^{i} \prod_{j=1}^{i} B_{p_{1}}\left(x, q^{j}\right)=F_{p}(x, q) .
$$

Indeed, each permutation, counted by $F_{p}(x, q)$ on the right hand side, either avoids $X$, which is counted by $B(x)$ on the left hand side, or contains at least one occurrence of $X$. In the latter case, suppose $a_{1}, a_{2}, \ldots, a_{i}$ are all occurrences of $X$ in a permutation as shown in Figure 9, so all the boxes $A_{j}$, where $0 \leq j \leq i$, are $X$-avoiding. Our key observation is that any occurrence of $p_{1}$ in a box $A_{j}$, where $1 \leq j \leq i$, together with each of the $a_{k}$, where $1 \leq k \leq j$, contribute an occurrence of $p$. Thus, the contribution of $A_{j}$ is $F_{p_{1}}\left(x, q^{j}\right)$ for $1 \leq j \leq i$, and the contribution of $A_{0}$ is $B(x)$. Finally, $x^{i}$ is given by $a_{i}$ 's and we can sum over all $i \geq 1$. This completes the proof of (18). Substituting the formulas of $B(x)$ and $B_{p_{1}}(x, q)$ into (18) gives the formula of $F_{p}(x, q)$. This completes the proof.

Note that $p_{1}$ in Theorem 4.1 must be of length $\geq 2$ because the result does not work for $p_{1}=\downarrow$. In the latter case though we deal with the pattern Nr. $16=! !$, whose avoidance and distribution are solved in [10]. 


\subsection{The pattern Nr. 33}

In our next theorem we obtain a generalization of the pattern Nr. $33=\stackrel{\bullet}{\bullet}$.

Theorem 4.2. Suppose that $p$ is the pattern shown in Figure 10, where $p_{1}$ is an irreducible mesh pattern of length $\geq 2$, and the labels $a, b, A$, and $B$ are to be ignored. Then,

$$
\begin{aligned}
& A_{p}(x)=\frac{\left(1+x^{2} A_{p_{1}}(x)\right) F(x)}{1+x^{2} F(x)}, \\
& F_{p}(x, q)=\frac{F(x)}{1+x F(x)}+\left(\frac{F(x)}{1+x F(x)}\right)^{2}\left(x+\sum_{i \geq 2} x^{i} \prod_{j=2}^{i} \frac{F_{p_{1}}\left(x, q^{\left(\begin{array}{l}
j \\
2
\end{array}\right)}\right)}{1+x F_{p_{1}}\left(x, q^{\left(\begin{array}{l}
j \\
2
\end{array}\right)}\right)}\right) \text {. } \\
& \begin{array}{l|l|l} 
& b & p_{1} \\
\hline a & B & \\
\hline A & &
\end{array}
\end{aligned}
$$

Figure 10: Related to the proof of Theorem 4.2

Proof. The case of avoidance is similar to our considerations of the pattern $X=\downarrow$. We assume that the elements $a$ and $b$ in an occurrence of $p$ in Figure 10 are leftmost possible. But then, the boxes $A$ and $B$ will be $X$-avoiding. Thus, we have the following functional equation:

$$
A_{p}(x)+x^{2} B^{2}(x)\left(F(x)-A_{p_{1}}(x)\right)=F(x) .
$$

Substituting (14) for $B(x)$ into (19), we obtain the formula of $A_{p}(x)$.

For the distribution, we have the following functional equation:

$$
B(x)+x B^{2}(x)+B^{2}(x) \sum_{i \geq 2} x^{i} \prod_{j=2}^{i} B_{p_{1}}\left(x, q^{\left(\begin{array}{c}
j \\
2
\end{array}\right)}\right)=F_{p}(x, q),
$$

where $B_{p_{1}}(x, q)$ is given in (17). Our proof of (20) is essentially the same as that of (18) and we omit it. The only difference is that here we have two 
$X$-avoiding boxes $A$ and $B$ resulting in the factor of $B^{2}(x)$ instead of $B(x)$. Substituting (17) for $B_{p_{1}}(x, q)$ into (20), we obtain the desired formula of $F_{p}(x, q)$, and thus complete the proof.

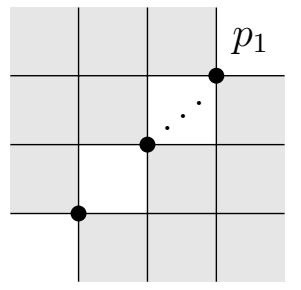

Figure 11: Related to Theorem 4.3

The proof of the following theorem follows similar steps to those in Theorem 4.2, and thus is omitted. We note that Theorem 4.3 is a far-reaching generalization of Theorem 4.2.

Theorem 4.3. Suppose that $p$ is the pattern shown in Figure 11, where $p_{1}$ is an irreducible mesh pattern. Let pattern $X=\bullet-$. Then the distribution of $p$ is

$F_{p}(x, q)=\sum_{i=1}^{k} x^{i-1}\left(\frac{F(x)}{1+x F(x)}\right)^{i}+\left(\frac{F(x)}{1+x F(x)}\right)^{k} \sum_{i \geq k} x^{i} \prod_{j=k}^{i} \frac{F_{p_{1}}\left(x, q^{\left(\begin{array}{l}j \\ k\end{array}\right)}\right)}{1+x F_{p_{1}}\left(x, q^{\left(\begin{array}{l}j \\ k\end{array}\right)}\right)}$.

\section{$5 \quad$ Remaining avoidance cases}

In this section, we study avoidance of generalizations of the patterns:

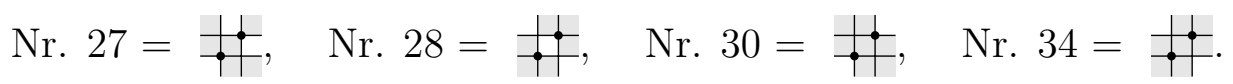

\subsection{Pattern Nr. 28}

The following theorem allows us to generalize the known avoidance result [10] for the pattern Nr. $28=\div$ by inserting two mesh patterns, $p_{1}$ and $p_{2}$, into it. We were unable to find the distribution of the pattern in the next theorem because of difficulties of controlling occurrences of the patterns $p$ and $p_{2}$, at the same time, in the rightmost bottom box. 
Theorem 5.1. Let $p$ be the pattern shown in Figure 12, where the labels a, $b$, and $A$ are to be ignored, and $p_{1}$ and $p_{2}$ are any mesh patterns. Then, the avoidance of $p$ is given by

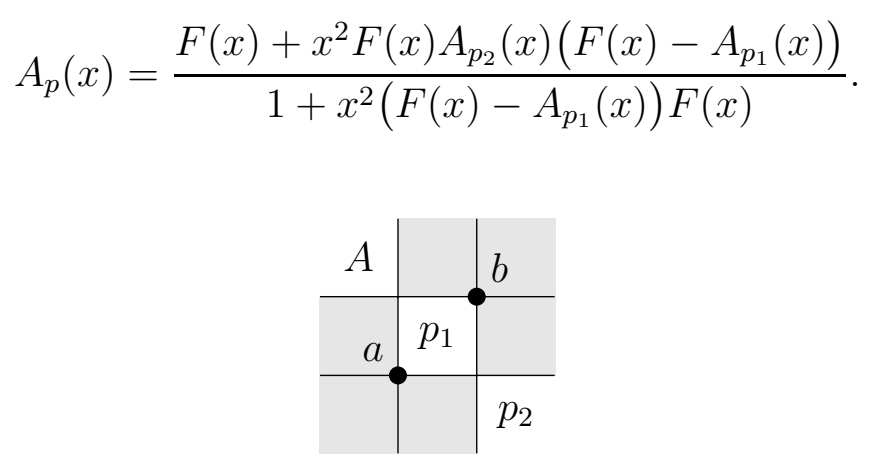

Figure 12: Related to the proof of Theorem 5.1

Proof. Let $A(x)$ be the generating function for the number of permutations avoiding the pattern $\frac{f_{i}}{p_{1}}$. Then, it follows from Theorem 3.5 that

$$
A(x)=\frac{F(x)}{1+x^{2}\left(F(x)-A_{p_{1}}(x)\right) F(x)} .
$$

We have the following functional equation:

$$
A_{p}(x)+x^{2} A(x)\left(F(x)-A_{p_{1}}(x)\right)\left(F(x)-A_{p_{2}}(x)\right)=F(x) .
$$

Indeed, each permutation, counted by $F(x)$ on the right hand side, either avoids $p$, counted by $A_{p}(x)$, or contains at least one occurrence of $p$. In the latter case, among all such occurrences, we can pick the occurrence $a b$ with the leftmost possible $a$ as shown in Figure 12. Referring to this figure, we note that the central box must contain at least one occurrence of $p_{1}$, which is counted by $F(x)-A_{p_{1}}(x)$, and the rightmost bottom box must contain at least one occurrence of $p_{2}$, counted by $F(x)-A_{p_{2}}(x)$. Moreover, the permutation in box $A$ must avoid the pattern $\frac{p_{i}}{p_{1}}$, which is counted by $A(x)$, since $a$ is the leftmost possible element in an occurrence of $p$. Finally, $a$ and $b$ contribute the factor of $x^{2}$. This completes our proof of (21) and hence gives the formula of $A_{p}(x)$ as desired. 
Remark 5.2. Note that exactly the same enumeration result as that in Theorem 5.1 holds for the pattern $\frac{p_{2} \mid p_{1} \cdot-}{p_{1}}$. Indeed, in the proof of Theorem 5.1 one just essentially need to substitute the "leftmost a" by the "rightmost b".

\subsection{Pattern Nr. 30}

We next consider generalizations of the pattern Nr. $30=\stackrel{\bullet}{\bullet}$.

Lemma 5.3 ([10, Theorem 3.5]). Let $p=\biguplus_{\dagger}, F(x, q)=\sum_{n \geq 0} x^{n} \sum_{\pi \in S_{n}} q^{p(\pi)}$, and $A(x)$ be the generating function for $S(p)$. Then,

$$
A(x)=\frac{(1+x) F(x)}{1+x+x^{2} F(x)}, \quad F(x, q)=\frac{(1+x-q x) F(x)}{1+(1-q) x+(1-q) x^{2} F(x)} .
$$

The avoidance of the patterns in the next two theorems is based on Lemma 5.3, but we were not able to derive the distribution of these patterns extending the respective formula in Lemma 5.3.

Theorem 5.4. Suppose that $p$ is the pattern shown in Figure 13, where $p_{1}$ is a mesh pattern with the leftmost bottom box non-shaded, and the labels a, $b$, and $A$ are to be ignored. Then, the avoidance of $p$ is given by

$$
A_{p}(x)=\frac{(1+x) F(x)+x^{2} A_{p_{1}}(x)}{1+x+x^{2} F(x)} .
$$

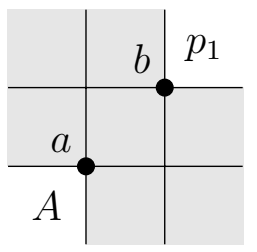

Figure 13: Related to the proof of Theorem 5.4

Proof. We have the following functional equation

$$
A_{p}(x)+\frac{x^{2} F(x)}{1+x+x^{2} F(x)}\left(F(x)-A_{p_{1}}(x)\right)=F(x) .
$$


Indeed, the right hand side counts all permutations, and the left hand side considers separately permutations avoiding $p$, counted by the $A_{p}(x)$ term in (22), and those containing at least one occurrence of $p$. In the latter case, among all such occurrences, we can pick the occurrence $a b$ with the leftmost possible $a$ as shown in Figure 13. Referring to this figure, we note that the North East box must contain at least one occurrence of $p_{1}$, counted by $F(x)-A_{p_{1}}(x)$. Moreover, a permutation in box $A$ must avoid both patterns $\because$ and $\bullet$, since $a$ is the leftmost possible. We denote by $B(x)$ the generating function of such permutations. Then, we have that

$$
B(x)+x B(x)=\frac{(1+x) F(x)}{1+x+x^{2} F(x)}
$$

by dividing the $\leftrightarrows$-avoiding permutations, whose enumeration is given by Lemma 5.3, into two parts depending on whether they avoid $\rightarrow$. Note that when a permutation contains the pattern $\downarrow \downarrow$, the sub-permutation consisting of the first $n-1$ positions avoids both patterns $\leftrightarrows$ and $\bullet$. Therefore, we get that

$$
B(x)=\frac{F(x)}{1+x+x^{2} F(x)} .
$$

Finally, $a$ and $b$ contribute the factor of $x^{2}$. Substituting the formula of $B(x)$ into (22), we obtain the desired formula of $A_{p}(x)$. This completes the proof.

\subsection{Pattern Nr. 27}

We next consider avoidance of a generalization of the pattern Nr. $27=$

Theorem 5.5. Suppose that $p$ is the pattern shown in Figure 14, where $p_{1}$ and $p_{2}$ are any mesh patterns, and the labels $a, b$, and $A$ are to be ignored. Then, the avoidance of $p$ is given by

$$
A_{p}(x)=F(x)-x^{2} \frac{F(x)}{1+x F(x)}\left(F(x)-A_{p_{1}}(x)\right)\left(F(x)-A_{p_{2}}(x)\right) .
$$




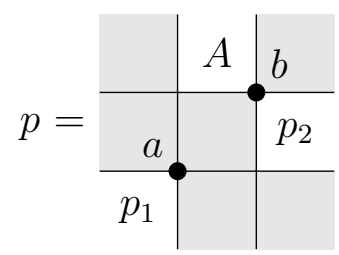

Figure 14: Related to the proof of Theorem 5.5

Proof. We have the following functional equation:

$$
A_{p}(x)+x^{2} B(x)\left(F(x)-A_{p_{1}}(x)\right)\left(F(x)-A_{p_{2}}(x)\right)=F(x),
$$

where $B(x)$ is the generating function for the number of $X$-avoiding permutations given in Theorem 1.1, which satisfies

$$
B(x)=\frac{F(x)}{1+x F(x)} .
$$

Indeed, $F(x)$ on the right hand side of (23) counts all permutations. On the left hand side of (23), we count separately permutations avoiding $p$, counted by the $A_{p}(x)$ term, and those containing at least one occurrence of $p$. In the latter case, among all occurrences of $p$, we pick the occurrence $a b$ with the leftmost possible $b$ as shown in Figure 14 and $a$ is then uniquely determined. Referring to this figure, we note that the South West box must contain at least one occurrence of $p_{1}$, counted by $F(x)-A_{p_{1}}(x)$, and the East box must contain at least one occurrence of $p_{2}$, counted by $F(x)-A_{p_{2}}(x)$. Moreover, the permutation in the box $A$ must avoid the pattern $X$, counted by $B(x)$, since $b$ is the leftmost possible. Finally, $a$ and $b$ contribute the factor of $x^{2}$. Thus, this completes the proof of (23). Substituting the formula of $B(x)$ into (23), we obtain the desired formula of $A_{p}(x)$.

Theorem 5.5 generalizes the avoidance of the pattern Nr. 27. However, generalizing its distribution is hard, because we need to control at the same time occurrences of the patterns $p$ and $Z=-\downarrow$ in the box $A$. Moreover, we cannot further generalize Theorem 5.5 by placing a mesh pattern $p_{3}$ in the box $A$, because $A$ must avoid $Z$ when requiring from $b$ to be the leftmost, so we will be forced to control two patterns $p_{3}$ and $Z$ at the same time. Of course, we can require from $b$ to be the rightmost, but then we will be forced to control two patterns in the East box. Finally, swapping $A$ and $p_{2}$ 
in Theorem 5.5 leads to the same enumeration result, which is not hard to see.

\subsection{Pattern Nr. 34}

We next consider generalizations of the pattern Nr. $34=\stackrel{!}{\rightarrow}$.

Lemma 5.6 ([10, Theorem 3.7]). Let $p=\biguplus !$. Then, the avoidance and distribution of $p$ are

$$
A_{p}(x)=\frac{F(x)}{1+x^{2} F(x)}, \quad F_{p}(x, q)=\frac{F(x)}{1+(1-q) x^{2} F(x)} .
$$

Replacing the two elements in the pattern Nr. 34 by the pattern $1 p_{1}$, where $p_{1}$ is any permutation of $\{2,3, \ldots, k\}, k \geq 2$, with all boxes shaded as in Figure 15, we can apply essentially the same arguments as in the proof of Lemma 5.6 in [10] to obtain the following theorem.

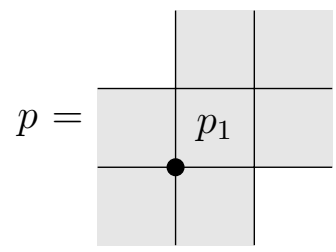

Figure 15: Related to Theorem 5.7

Theorem 5.7. Suppose that $p$ is the pattern shown in Figure 15, where $k \geq 1$ elements are in increasing order in the middle box. Then, the avoidance and distribution of $p$ are given by

$$
A_{p}(x)=\frac{F(x)}{1+x^{k} F(x)}, \quad F(x, q)=\frac{F(x)}{1+(1-q) x^{k} F(x)} .
$$

The avoidance of the pattern Nr. 34 can be generalized, which is done in the next theorem, but the distribution is hard because we need to control $p_{2}$ and $p$ in the same box in that theorem.

Theorem 5.8. Suppose that $p$ is the pattern shown in Figure 16, where $p_{1}$ is any permutation of $\{2,3, \ldots, k\}, k \geq 1$, with all boxes shaded, $p_{2}$ is any 
mesh pattern, and the labels $a$ and $A$ are to be ignored. Then, the avoidance of $p$ is given by

$$
\begin{array}{r}
A_{p}(x)=F(x)-\frac{x^{k} F(x)}{1+x^{k} F(x)}\left(F(x)-A_{p_{2}}(x)\right) . \\
p=\begin{array}{l|l|l}
A & & \\
\hline a_{0} & p_{1} & \\
\hline & & p_{2}
\end{array}
\end{array}
$$

Figure 16: Related to the proof of Theorem 5.8

Proof. Let $D(x)$ be the generating function for the number of permutations avoiding the pattern in Figure 15. Then, it follows from Theorem 5.7 that

$$
D(x)=\frac{F(x)}{1+x^{k} F(x)} .
$$

We have the following functional equation:

$$
A_{p}(x)+x^{k} D(x)\left(F(x)-A_{p_{2}}(x)\right)=F(x) .
$$

Indeed, the right hand side counts all permutations. On the left hand side, we count separately permutations avoiding $p$, counted by $A_{p}(x)$, and those containing at least one occurrence of $p$. In the latter case, among all occurrences of $p$, we can pick the occurrence with the leftmost possible $a$ as shown in Figure 16. Referring to this figure, we note that the South East box must contain at least one occurrence of $p_{2}$, counted by $F(x)-A_{p_{2}}(x)$. Moreover, the permutation in box $A$ must avoid the pattern in Figure 15 that is counted by $D(x)$, since $a$ is the leftmost possible. There are no other restrictions on $A$ because the pattern in Figure 15 cannot begin in $A$ and end somewhere else. Finally, the $k$ elements in the middle box contribute the factor of $x^{k}$. Thus, by combing with the formula of $D(x)$, we complete the proof of (24), and hence give the formula of $A_{p}(x)$. 


\section{Concluding remarks}

We have a number of general results related to distribution or avoidance of several infinite families of mesh patterns. How to describe the class of mesh patterns for which our distribution or avoidance results can be applicable? Namely, in which situations one can break the problem of enumerating mesh patterns into smaller problems using our theorems? What is the complexity of recognizing the class?

\section{Acknowledgments}

The first author is grateful to the administration of the Center for Combinatorics at Nankai University for their hospitality during the author's stay in April 2018. The second author was partially supported by the National Science Foundation of China (Nos. 11701424).

\section{References}

[1] S. Avgustinovich, S. Kitaev, and A. Valyuzhenich, Avoidance of boxed mesh patterns on permutations, Discrete Appl. Math., 161 (2013), 4341.

[2] N. Borie, On the combinatorics of quadrant marked mesh patterns in 132-avoiding permutations, Australasian J. Combin., 64(1) (2016), 140153.

[3] P. Brändén and A. Claesson, Mesh patterns and the expansion of permutation statistics as sums of permutation patterns, Electronic J. Combin., 18(2) (2011), \#P5.

[4] I. Hilmarsson, I. Jónsdóttir, S. Sigurdardóttir, L. Vidarsdóttir, and H. Ulfarsson, Wilf-classification of mesh patterns of short length, Electr. J. Combin., 22(4) (2015), \#P4.13.

[5] M. Jones, S. Kitaev and J. Remmel, Frame patterns in $n$-cycles, Discr. Math., 338 (2015), 1197-1215.

[6] S. Kitaev, Patterns in permutations and words, Springer, 2011. 
[7] S. Kitaev and J. Liese, Harmonic numbers, Catalan's triangle and mesh patterns, Discrete Math., 313 (2013), 1515-1531.

[8] S. Kitaev and J. Remmel, Quadrant marked mesh patterns in alternating permutations, Séminaire Lotharingien de Combinatoire, B68a (2012).

[9] S. Kitaev, J. Remmel and M. Tiefenbruck, Quadrant marked mesh patterns in 132-avoiding permutations, Pure Mathematics and Applications (Pu.M.A.), 23(3) (2012), 219-256.

[10] S. Kitaev, P. B. Zhang, Distributions of mesh patterns of short lengths, arXiv:1811.07679.

[11] J. Liese, J. Remmel, Q-analogues of the number of permutations with k-excedances, Pure Mathematics and Applications, 21(2) (2010), 285320 .

[12] R. P. Stanley. Enumerative combinatorics. Vol. 1, volume 49 of Cambridge Studies in Advanced Mathematics. Cambridge University Press, Cambridge, 1997.

[13] B. E. Tenner, Coincidental pattern avoidance, J. Combin., 4 (2013), $311-326$.

[14] B. E. Tenner, Mesh patterns with superfluous mesh, Adv. Appl. Math., 51 (2013), 606-618. 